\title{
Combining Thin-Section Coronal and Axial Diffusion Weighted Imaging: Good Practice in Middle Ear Cholesteatoma Neuroimaging
}

\author{
Camilla Russo ${ }^{1}$, Antonella Miriam Di Lullo ${ }^{2,3}$, Elena Cantone ${ }^{2}$, Michele Klain ${ }^{1}$, \\ Gaetano Motta ${ }^{4}$, Andrea Elefante ${ }^{1}$ and Michele Cavaliere ${ }^{2 *}$ \\ ${ }^{1}$ Dipartimento di Scienze Biomediche Avanzate - Università degli Studi di Napoli "Federico II", Naples, Italy, ${ }^{2}$ Dipartimento di \\ Neuroscienze, Scienze Riproduttive e Odontostomatologiche - Università degli Studi di Napoli "Federico II", Naples, Italy, \\ ${ }^{3}$ CEINGE - Advanced Biotechnology, Naples, Italy, ${ }^{4}$ Dipartimento di Scienze Anestesiologiche, Chirurgiche e dell'Emergenza \\ - Università degli Studi della Campania "Luigi Vanvitelli”, Naples, Italy
}

Keywords: middle ear, cholesteatoma, magnetic resonance imaging, diffusion weighted imaging DWI, fusion imaging

\section{INTRODUCTION}

OPEN ACCESS

Edited by:

Louis Murray Hofmeyr

Stellenbosch University, South Africa

Reviewed by:

Andrea Canale

University of Turin, Italy

André Burger,

Maine Radiology, South Africa

${ }^{*}$ Correspondence:

Michele Cavaliere michele.cavaliere@unina.it

Specialty section: This article was submitted to Neuro-Otology, a section of the journal

Frontiers in Neurology

Received: 15 September 2020

Accepted: 02 August 2021

Published: 07 September 2021

Citation:

Russo C, Di Lullo AM, Cantone E, Klain M, Motta G, Elefante $A$ and

Cavaliere M (2021) Combining

Thin-Section Coronal and Axial

Diffusion Weighted Imaging: Good

Practice in Middle Ear Cholesteatoma

Neuroimaging.

Front. Neurol. 12:606692.

doi: 10.3389/fneur.2021.606692
Along with clinical and otoscopic examination, magnetic resonance imaging (MRI) was proved to be the golden standard to assess the presence of cholesteatoma within temporal bone, both in middle ear cavity and mastoid. In particular, due to its specific composition (a cystic keratin-filled core surrounded by stratified squamous epithelium), cholesteatoma can be easily documented on diffusion-weighted imaging (DWI) as an area of striking hyperintensity due to restricted water diffusion $(1,2)$. Indeed, although computed tomography (CT) better defines localization and extent of the inflammatory tissue as well as the possible presence of bone erosions, only MR-DWI is able to define the nature of middle ear cavity opacification (cholesteatoma vs. granulation tissue) (3). Moreover, in recent times the extrapolation of quantitative values on apparent diffusion coefficient (ADC) maps generated from DWI has been proposed as a tool for distinguishing cholesteatoma from other types of middle ear inflammatory disorders (i.e., non-cholesteatomatous granulation tissue and abscesses), as well as to assess the risk of recurrence after surgical removal (4-7).

However, when referring to DWI for the assessment of skull base disorders such as cholesteatoma, it should be noted that a variety of different techniques could be applied (ranging from traditional spin-echo echo-planar images-EPI-to the more recently developed fast spin-echo-based non-EPI). Although these techniques are based on similar diffusion encoding, non-EPI ones differ in terms of image acquisition allowing for higher spatial resolution and lower susceptibility artifacts at air-bone interfaces (8-15).

Indeed, MRI protocol for hearing loss and to rule out the presence of cholesteatoma is generally based on the combination of sequences for brain imaging (generally including standard axial DWI, by far the most frequently used is spin-echo echo-planar due to its short imaging time and good contrast resolution) and specific sequences for the temporal bone (including thin-section coronal fast spin-echo-based non-EPI DWI, with some differences across sequences depending on MR unit vendor) (16); these latter are always acquired with small field of view (FOV), maximum section width of $3 \mathrm{~mm}$ and minimal or no inter-slice gap (1).

With this background, the aim of this retrospective study is to critically revise the role of combined thin-section coronal fast spin-echo-based non-EPI DWI of the temporal bone and axial spin-echo echo-planar DWI for the detection of middle ear cholesteatoma (both in the setting of acquired and residual/recurrent disease), in order to improve clinical management and optimize surgical procedures $(17,18)$. 


\section{MATERIALS AND METHODS}

We retrospectively analyzed all patients with clinical suspicion of unilateral middle ear cholesteatoma who underwent MRI at our University Department between January 2010 and January 2020; both acquired and residual/recurrent disease were considered for our purposes, whereas no case of congenital cholesteatoma was included in the study. Cholesteatoma diagnosis was then confirmed at surgery. MRI was performed on the same $1.5 \mathrm{~T}$ unit (Philips Intera, Philips Medical Systems, Netherlands) with an 8channel head coil. MRI protocol for hearing loss was based on sequences for whole brain imaging (generally including standard axial DWI) and specific sequences for the temporal bone (generally including thin-section coronal DWI). Standard MRI examination did not routinely include intravenous injection of gadolinium-based contrast media; patients with hearing implants and motion artifacts at MRI examination were excluded from the analysis.

DWI sequences parameters were set as follow:

- Brain axial spin-echo EPI DWI: 24 slides; TR 2,800 ms; TE $75 \mathrm{~ms}$; thickness $5.00 \mathrm{~mm}$; inter-slices gap $5 \mathrm{~mm}$; FA 90; view size $2,338 \times 1,228$; matrix $128 \times 128 ; b=0, b=500$, and $b=$ $1,000 \mathrm{~s} / \mathrm{mm}^{2} ; 4$ averages;

- Thin-section coronal multi-shot (MSH) non-EPI DWI of the temporal bone: 20 slides; TR 3,000 ms; TE $82.44 \mathrm{~ms}$; thickness $3.00 \mathrm{~mm}$; inter-slices gap 0; FA 90; view size 1,168 $\times 1,230$; matrix $152 \times 152 ; b=0$ and $b=800 \mathrm{~s} / \mathrm{mm}^{2} ; 5$ averages; cardiac gating to limit patient-related artifacts due to heart pulse and blood flow.

Finally, 173 MRI examinations were reviewed (97 female; 76 males; mean age $44.3 \mathrm{y}$; range $18-83 \mathrm{y}$ ), of them 89 for newly diagnosed acquired middle ear cholesteatoma (51.5\%) and 84 for residual/recurrent disease $(48.5 \%)$. Few patients $(n=9)$ had only axial DWI, as cholesteatoma discovery was an incidental finding on brain MRI performed for different diagnostic purpose; of the remaining subjects, 62 patients had only thin-section coronal DWI, whereas 102 had both axial and thin-section coronal DWI. Therefore, 111 axial DWI (54 newly diagnosed acquired and 57 residual/recurrent cholesteatomas) and 164 thin-section coronal DWI (80 newly diagnosed acquired and 84 residual/recurrent cholesteatomas) were globally revised; localizations of restricted water diffusion areas suggestive for cholesteatoma were noted separately on both axial and thin-section coronal DWI by two experienced neuroradiologists in consensus.

For thin-section coronal non-EPI DWI, axial EPI DWI, and combined thin-section coronal and axial DWI, diagnostic accuracy (DA), sensitivity, specificity, negative predictive value (NPV) and positive predictive value (PPV) were computed. All statistical analysis was performed using XLSTAT software (v.2019.1).

\footnotetext{
Abbreviations: MRI, Magnetic Resonance Imaging; DWI, Diffusion Weighted Imaging; CT, Computed Tomography; ADC, Apparent Diffusion Coefficient; EPI, Echo-Planar Imaging; FOV, Field Of View; DA, Diagnostic Accuracy; NPV, Negative Predictive Value; PPV, Positive Predictive Value; SSH, Single-Shot; TSE, Turbo Spin-Echo; MSH, Multi-Shot.
}

\section{RESULTS}

Standard axial EPI DWI alone showed an overall DA of 0.66 in identifying cholesteatomatous tissue within middle ear cavity, with 0.79 sensitivity, 0.32 specificity, $0.75 \mathrm{PPV}$ and $0.37 \mathrm{NPV}$.

Thin-section coronal non-EPI DWI of the temporal bone had an overall DA of 0.73 , with 0.83 sensitivity, 0.60 specificity, 0.71 PPV, and $0.75 \mathrm{NPV}$. Finally, combining axial brain EPI DWI and thin-section coronal non-EPI DWI of the temporal bone, DA increased up to 0.94 , with 0.98 sensitivity, 0.94 specificity, 0.98 $\mathrm{PPV}$, and 0.94 NPV.

Moreover, when separately analyzing results from the subgroups, it was found that the impact of the combination of the two acquisition plans was higher for residual/recurrent disease rather than for primary acquired middle ear cholesteatoma. Indeed, for primary acquired cholesteatoma standard axial EPI DWI showed a DA of 0.71 whereas thin-section coronal non-EPI DWI showed a DA of 0.88 ; combining the two acquisition techniques, DA increased to 0.98 . Conversely, for recurrent/residual disease standard axial EPI DWI showed a DA of 0.52 and thin-section coronal non-EPI DWI showed a DA of 0.68 , whereas with the combination of the two acquisition techniques DA increased to 0.90 . The magnitude of the improvement was therefore higher in the second group compared to the first one.

Inter-observer reliability regarding cholesteatomas' identification, assessed with Cohen's kappa, was found at 0.94 .

Overall results in terms of diagnostic testing accuracy are listed in Table 1. An example of axial and coronal DWI, along with fusion imaging with ultra-thin heavily-weighted 3D T2w sequences to allow for a better localization of cholesteatomatous tissue, is shown in Figure 1.

\section{DISCUSSION}

DWI has become a pivotal MRI technique for the detection of middle ear cholesteatoma, both in case of newly diagnosed acquired lesions and post-surgical evidence of residual/recurrent disease at longitudinal follow-up $(10,19)$. In particular, thin-section coronal fast spin-echo-based non-EPI techniques have largely replaced traditional spin-echo EPI techniques for temporal bone imaging, due to their better spatial resolution with different acquisition matrix, lower signal-to-noise ratio, and reduced susceptibility artifacts at air-bone interfaces, thus allowing for cholesteatoma detection even when smaller than

TABLE 1 | Table resuming diagnostic testing accuracy (DA) for all cholesteatomas (first column), primary acquired cholesteatomas (second column), and residual/recurrent cholesteatomas (third column).

\begin{tabular}{lccc}
\hline & $\begin{array}{c}\text { All } \\
\text { cholesteatomas }\end{array}$ & $\begin{array}{c}\text { Primary } \\
\text { acquired c. }\end{array}$ & $\begin{array}{c}\text { Residual/ } \\
\text { recurrent c. }\end{array}$ \\
\hline Axial EPI DWI & 0.66 & 0.71 & 0.52 \\
Coronal non-EPI DWI & 0.73 & 0.88 & 0.68 \\
Combined DWIs & 0.94 & 0.98 & 0.90
\end{tabular}




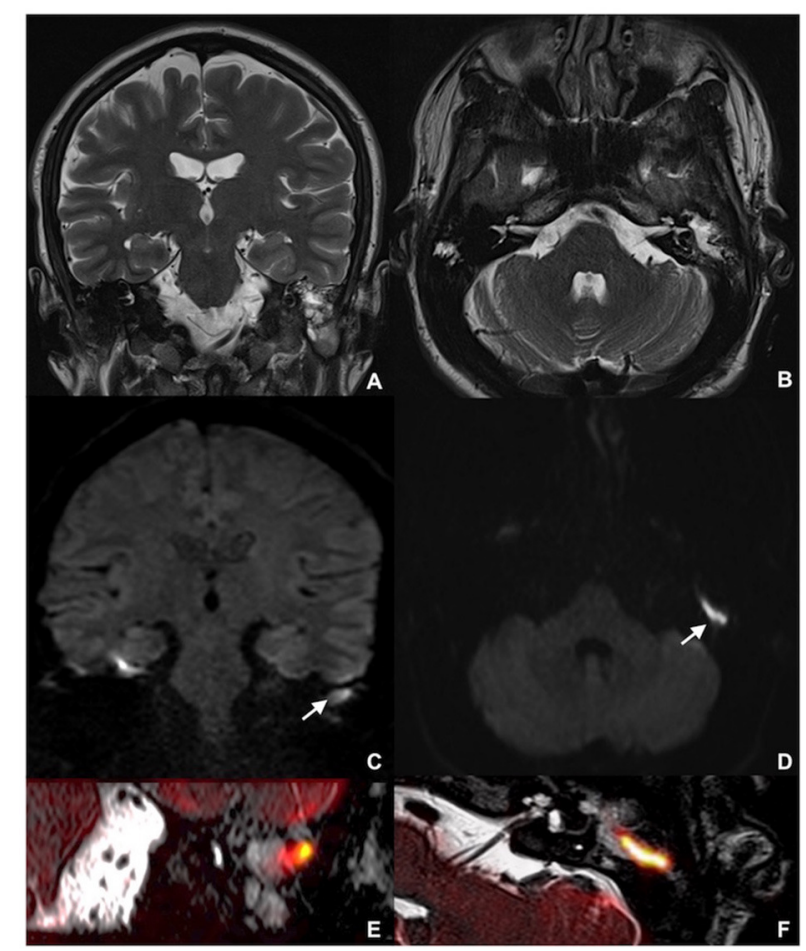

FIGURE 1 | MRI showing left middle ear recurrent cholesteatoma in a 26-year-old man: coronal (A) and axial (B) TSE T2w; coronal MSH non-EPI DWI (C) and axial SE-EPI DWI (D); detailed coronal (E) and axial (F) fusion imaging of both ultra-thin heavily-weighted 3D T2w sequences and DWI images. MRI, magnetic resonance imaging; TSE, turbo spin echo; $\mathrm{MSH}$, multi-shot; EPI, echo planar imaging; DWI, diffusion weighted imaging; SE, spin-echo.

$5 \mathrm{~mm}(9,20-22)$. These sequences include a variety of different techniques depending on MR vendor, ranging from halffourier acquisition single-shot turbo spin-echo (SSH-TSE) to PROPELLER DWI, from BLADE to MSH non-EPI DWI; among them, the latter has been adopted in our MRI protocol for temporal bone as previously described $(1,8)$.

However, despite the good resolution and the lower incidence of interface artifacts in thin-section coronal fast spin-echo-based

\section{REFERENCES}

1. Más-estellés F, Mateos-fernández M, Carrascosa-Bisquert B, Facal de Castro F, Puchades- Román I, Morera-Pérez C. Contemporary non-echo-planar diffusion-weighted imaging of middle ear cholesteatomas. RadioGraphics. (2012) 32:1197-213. doi: 10.1148/rg.324115109

2. Rutkowska J, Özgirgin N, Olszewska E. Cholesteatoma definition and classification: a literature review. J Int Adv Otol. (2017) 13:266-71. doi: 10.5152/iao.2017.3411

3. Barath K, Huber AM, Stampfli P, Varga Z, Kollias S. Neuroradiology of cholesteatomas. Am J Neuroradiol. (2011) 32:221-9. doi: 10.3174/ajnr.A2052

4. Russo C, Elefante A, Di Lullo AM, Carotenuto B, D’Amico A, Cavaliere $\mathrm{M}$, et al. ADC benchmark range for correct diagnosis of primary and recurrent middle ear cholesteatoma. Biomed Res Int. (2018) 2018:16. doi: $10.1155 / 2018 / 7945482$
non-EPI techniques, few cases of false negatives and false positives have still been observed. While false negatives are mainly due to very small lesion size, false positives can be due to several different condition that could enhance the presence of MRI artifacts (i.e., presence of prosthetic materials or metallic dental implants, recent surgery, admixed otomastoiditis with areas of abscessualisation) $(1,23,24)$. To overcome possible pitfalls and implement the interpretation of indeterminate singleplan DWI, additional axial DWI can be a worthwhile information support. In our experience, combining thin-section coronal nonEPI DWI and axial EPI-DWI improved the identification of small middle ear lesion, particularly in the setting of post-operative residual/recurrent disease where potential confounding factors can make proper and timely diagnosis more challenging.

Main limitations of the study are represented by the retrospective design and the moderate sample size; moreover, although similar diagnostic performances of different fast spinecho-based non-EPI techniques were reported in literature, it should be considered that these observations apply to a specific non-EPI sequence and a single MR unit (despite probably representing the most common setting in daily clinical routine).

In conclusion, standard axial EPI-DWI combined to thinsection coronal fast spin-echo-based non-EPI DWI of the temporal bone has the potential to improve the diagnosis of middle ear cholesteatoma. This is all the more true when considering lesions smaller than $5 \mathrm{~mm}$ and/or residual/recurrent disease, when single plan DWI can be misleading and inconclusive. With this knowledge and due to its short acquisition time, we strongly recommend always including standard axial brain EPI-DWI along with thin-section coronal DWI in case of suspected cholesteatoma, in order to improve observers' confidence and enhance lesions' detection.

\section{AUTHOR CONTRIBUTIONS}

$\mathrm{CR}$ and $\mathrm{AE}$ conceived the study. AMDL and MC performed the analysis. GM and EC verified the analytical methods. MK supervised the findings of this work. All authors discussed the results and contributed to the final manuscript. 
9. Elefante A, Cavaliere M, Russo C, Caliendo G, Marseglia M, Cicala D, et al. Diffusion weighted MR imaging of primary and recurrent middle ear cholesteatoma: an assessment by readers with different expertise. Biomed Res Int. (2015) 2015:597896. doi: 10.1155/2015/597896

10. De Foer B, Vercruysse J, Bernaerts A, Maes J, Deckers F, Michiels J, et al. The value of single-shot turbo spin-echo diffusion-weighted MR imaging in the detection of middle ear cholesteatoma. Neuroradiology. (2007) 49:8418. doi: 10.1007/s00234-007-0268-3

11. Yamashita K, Yoshiura T, Hiwatashi A, Kamano H, Dashjamts T, Shibata $\mathrm{S}$, et al. Detection of middle ear cholesteatoma by diffusion-weighted MR imaging: multishot echo-planar imaging compared with single-shot echoplanar imaging. Am J Neuroradiol. (2011) 32:1915-8. doi: 10.3174/ajnr.A2651

12. Dremmen MHG, Hofman PAM, Hof JR, Stokroos RJ, Postma AA. The diagnostic accuracy of non-echo-planar diffusion-weighted imaging in the detection of residual and/or recurrent cholesteatoma of the temporal bone. Am J Neuroradiol. (2012) 33:439-44. doi: 10.3174/ajnr.A2824

13. Lingam ÃRK, Bassett P. A meta-analysis on the diagnostic performance of non-echoplanar diffusion-weighted imaging in detecting middle ear cholesteatoma: 10 years on. Otol Neurotol. (2017) 38:521-8. doi: 10.1097/MAO.0000000000001353

14. Cavaliere M, Di Lullo AM, Cantone E, Scala G, Elefante A, Russo C, et al. Cholesteatoma vs granulation tissue: a differential diagnosis by DWI-MRI apparent diffusion coefficient. Eur Arch Otorhinolaryngol. (2018) 275:223743. doi: 10.1007/s00405-018-5082-5

15. Fischer N, Schartinger V, Dejaco D, Schmutzhard J, Riechelmann H, Plaikner M, et al. Readout-segmented echo-planar DWI for the detection of cholesteatomas: correlation with surgical validation. AJNR. (2019) 40:10559. doi: 10.3174/ajnr.A6079

16. Soni N, Gupta N, Kumar Y, Mangla M, Mangla R. Role of diffusion-weighted imaging in skull base lesions: a pictorial review. Neuroradiol J. (2017) 0:115. doi: 10.1177/1971400917709624

17. Li KL, Agarwal V, Moskowitz HS, Abuzeid WM. Surgical approaches to the petrous apex. World J Otorhinolaryngol Neck Surg. (2020) 6:10614. doi: 10.1016/j.wjorl.2019.11.002

18. Cavaliere M, Ricciardiello F, Iengo M, Mesolella M. Stapedotomy: functional results with different diameter prostheses. ORL. (2012) 74:93-6. doi: 10.1159/000335927

19. Lingam RK, Connor SEJ, Casselman JW, Beale T. MRI in otology : applications in cholesteatoma and Ménière's disease. Clin Radiol. (2017) 73:35-44. doi: 10.1016/j.crad.2017.09.002
20. Lingam RK, Nash R, Majithia A, Kalan A, Singh A. Non-echoplanar diffusion weighted imaging in the detection of post-operative middle ear cholesteatoma: navigating beyond the pitfalls to find the pearl. Insights Imaging. (2016) 7:669-78. doi: 10.1007/s13244-016-0516-3

21. Lingam RK, Khatri $P$, Hughes J, Singh A. Apparent diffusion coefficients for detection of postoperative middle ear cholesteatoma on non-echo-planar diffusion-weighted images. Radiology. (2013) 269:504-10. doi: 10.1148/radiol.13130065

22. van Egmond SL, Stegeman I, Grolman W, Aarts MCJ. A systematic review of non-echo planar diffusion-weighted magnetic resonance imaging for detection of primary and postoperative cholesteatoma. Otolaryngol Head Neck Surg. (2015) 154:233-40. doi: 10.1177/0194599815613073

23. Muhonen EG, Mahboubi H, Moshtaghi O, Sahyouni R, Ghavami Y, Maducdoc $\mathrm{M}$, et al. False-positive cholesteatomas on non-echoplanar diffusionweighted magnetic resonance imaging. Otol Neurotol. (2020) 41:e58892. doi: 10.1097/MAO.0000000000002606

24. Dadau C, Draper A, GkaGkanasiou M, Charles-Edwards G, Pai I, Connor S. Cholesteatoma: multishot echo-planar vs non echo- planar diffusion-weighted MRI for the prediction of middle ear and mastoid cholesteatoma. BJR Open. (2019) 1:20180015. doi: 10.1259/bjro.20 180015

Conflict of Interest: The authors declare that the research was conducted in the absence of any commercial or financial relationships that could be construed as a potential conflict of interest.

Publisher's Note: All claims expressed in this article are solely those of the authors and do not necessarily represent those of their affiliated organizations, or those of the publisher, the editors and the reviewers. Any product that may be evaluated in this article, or claim that may be made by its manufacturer, is not guaranteed or endorsed by the publisher.

Copyright (C) 2021 Russo, Di Lullo, Cantone, Klain, Motta, Elefante and Cavaliere. This is an open-access article distributed under the terms of the Creative Commons Attribution License (CC BY). The use, distribution or reproduction in other forums is permitted, provided the original author(s) and the copyright owner(s) are credited and that the original publication in this journal is cited, in accordance with accepted academic practice. No use, distribution or reproduction is permitted which does not comply with these terms. 